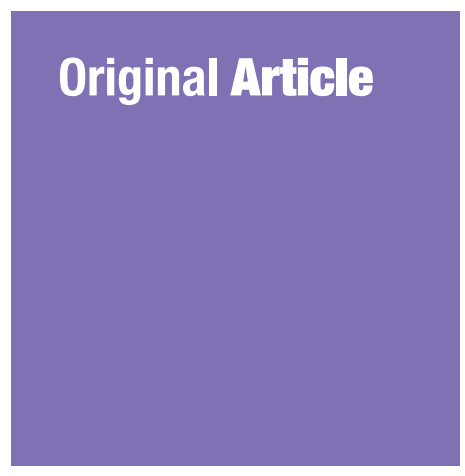

Submitted: 14 Jul 2016 Accepted: 19 Dec 2016 Online: 14 Apr 2017

\section{Attachment, Proliferation, and Morphological Properties of Human Dermal Fibroblasts on Ovine Tendon Collagen Scaffolds: A Comparative Study}

\author{
FAUZI Mh Busra1, Yogeswaran LoKanATHAN ${ }^{1}$, Masrina Mohd \\ NadzIR ${ }^{3}$, Aminuddin Saim ${ }^{1,2}$, Ruszymah Bt Hj Idrus ${ }^{1,4}$, Shiplu Roy \\ CHOWDHURY $^{1}$
}

$1 \quad$ Tissue Engineering Centre, UKM Medical Centre, Jalan Yaacob Latiff, Bandar Tun Razak, 56000 Cheras, Kuala Lumpur, Malaysia

2 Ear, Nose \& Throat Consultant Clinic, Ampang Puteri Specialist Hospital, Taman Dato Ahmad Razali, 68000 Ampang, Selangor, Malaysia

3 School of Chemical Engineering, Engineering Campus, Universiti Sains Malaysia, 14300 Nibong Tebal, Pulau Pinang, Malaysia

4 Department of Physiology, Faculty of Medicine, UKM Medical Centre, Jalan Yaacob Latiff, Bandar Tun Razak, 56000 Cheras, Kuala Lumpur, Malaysia 


\section{Introduction}

Tissue engineering has emerged as an alternative therapy for the development of artificial tissue in regenerative medicine. It involves the combination of three components: cells, scaffolds, and biomolecules (1). Scaffolds provide a temporary framework for $3 \mathrm{D}$ substitutes, with a microenvironment suitable for the proliferation and differentiation of cells, as well as the formation of an extracellular matrix (ECM) to generate target tissues (2-3). Given the variation in properties of the native matrix between tissue types, various types of scaffolds have been fabricated. These include film (by casting), sponge (by freeze-drying), hydrogel (by gelation), nanofibres (by electrospinning), and ceramic (by sintering) to mimic the native tissue framework (4-6). However, regardless of tissue type, several key factors must be considered to determine the suitability of the scaffold for tissue engineering. Biocompatibility and biodegradability are the basic criteria for a scaffold to be used in tissue engineering applications. Several factors including the topography, chemistry, microarchitecture, and mechanical properties of the scaffold, have been shown to influence the cell-scaffold interaction (7-8).

In our previous studies, collagen type I (col I) was extracted from ovine tendon and used to fabricate scaffolds such as sponge (9), and both aligned and random films (10). These scaffolds were shown to be biocompatible with human dermal fibroblasts (HDF) (9). They were also shown to regulate HDF behaviour, depending on scaffold type and characteristics. However, no comparative studies were performed. Thus, the objective of the current study was to compare the attachment, proliferative, and morphological properties of $\mathrm{HDF}$ on various ovine tendon collagen type I (OTC-I) scaffolds such as film, sponge, and hydrogel, with or without crosslinking. A polystyrene surface and OTC-I coated polystyrene surface (with or without crosslinking) were used to determine HDF properties in comparison with OTC-I scaffolds.

\section{Materials and Methods}

This study was conducted between 2014 and 2016 at the Tissue Engineering Centre, UKM Medical Centre. The research was approved by the UKM Research Ethics Committee under Project Code: FF-2015-087.
Collagen extraction and purification from ovine tendon

Collagen extraction and purification were performed as described elsewhere (10). The collected tendon was cleaned of fascia and debris prior to freeze-drying. The dried tendon was then dissolved in $0.35 \mathrm{M}$ acetic acid (AnalaR; VWR, USA), concentrated using sodium chloride ( $0.05 \mathrm{~g} / \mathrm{ml}$; Sigma, USA), and centrifuged. The resultant collagen precipitate was then dialysed for 72 hours using a dialysis tube (cut-off value of $14 \mathrm{kDa}$; Sigma), freeze-dried, and re-dissolved in acetic acid for further use.

\section{OTC-I-coated surface}

The polystyrene culture surface (12-well plate; Greiner BioOne, Germany) was coated with $10 \mu \mathrm{g} / \mathrm{cm}^{2}$ OTC-I solution and incubated at $37^{\circ} \mathrm{C}$ for 2 hours. The excess fluid was removed from the coated surface and dried overnight.

\section{Fabrication of OTC-I scaffolds}

OTC-I was used to fabricate three different scaffolds (area $\times$ thickness): film $\left(3.9 \mathrm{~cm}^{2} \times\right.$ $0.015 \mathrm{~cm})$, hydrogel $\left(3.9 \mathrm{~cm}^{2} \times 0.2 \mathrm{~cm}\right)$, and sponge $\left(3.9 \mathrm{~cm}^{2} \times 0.2 \mathrm{~cm}\right)$. Film was fabricated by air-drying the OTC-I solution at $37{ }^{\circ} \mathrm{C}$ for 2 hours, as described elsewhere (10). To fabricate hydrogel, the OTC-I solution was neutralised to $\mathrm{pH}$ 7.0 using $1 \mathrm{M}$ sodium hydroxide (Sigma) before gelation at $37{ }^{\circ} \mathrm{C}$ for 2 hours. The OTC-I sponge was prepared using the freeze-drying technique $(9,11)$. The OTC-I solution was poured into the desired mould and frozen at $-80{ }^{\circ} \mathrm{C}$ for 6 hours, followed by freeze-drying for 24 hours to 48 hours.

\section{Crosslinking of OTC-I}

Crosslinking of OTC-I-coated surface and OTC-I scaffolds was performed using genipin (GNP; CBC, Taiwan) as a natural crosslinker (12, 13). The coated surface and film were incubated with $0.1 \%(\mathrm{w} / \mathrm{v})$ GNP solution for 2 hours. The OTC-I hydrogel and sponge were incubated with GNP for 6 hours. Subsequently, all scaffolds and surfaces were washed with a large amount of phosphate buffer saline (PBS; Sigma, USA) to remove any residual GNP and byproducts. OTCI-coated surface and scaffolds were sterilised with $70 \%$ ethanol for 20 minutes and washed with PBS. Prior to cell culturing, they were washed with sterile PBS and incubated in a minimal medium without growth supplement for one hour. 


\section{Mechanical evaluation}

Mechanical testing was performed as described elsewhere (10). Briefly, OTC-I scaffolds were cut into $3 \mathrm{~cm}^{2}$ pieces and attached to an Instron 8874 (Instron, USA) using a clamp at both ends. A $50 \mathrm{~N}$ load transducer at a crosshead velocity of $0.05 \mathrm{~mm} / \mathrm{min}$ was used to evaluate their mechanical strength. The tensile strain and Young's modulus of the OTC-I scaffolds were measured.

\section{Human skin cell harvesting \& culturing}

Redundant skin tissues were obtained during abdominoplasty from six consented patients (25-40 years old; $N=6$ ) and processed within 24 hours (11). Skin samples were minced into small pieces and digested with $0.6 \%$ collagenase type I (Worthington, USA) for 5 hours followed by treatment with trypsinEDTA (Lonza, USA) for 10 minutes. Cells were suspended in co-culture medium, containing an equal mixture of Epilife (Gibco/BRL, USA) and F12:DMEM (1:1; Gibco/BRL) containing 10\% FBS (Biowest, USA) and seeded in three wells of a six-well culture plate (Greiner, Germany). This was maintained in an incubator at $37^{\circ} \mathrm{C}$ and $5 \%$ $\mathrm{CO}_{2}$ with changes in medium every 2 to 3 days. After reaching $80 \%$ confluence, the HDF was removed from the co-culture using differential trypsinisation (14) and seeded in a $75 \mathrm{~cm}^{2}$ culture vessel with F12:DMEM medium containing 10\% FBS. HDF $(N=6)$ at passage 3 was seeded on the different OTC scaffolds (technical replicates, $n=3)$ at a density of 4000 cells $/ \mathrm{cm}^{2}$ and cultured for 7 days. The viability of HDF was evaluated using MTT (3-(4,5-dimethylthiazol2-yl)-2,5-diphenyltetrazolium bromide) assay according to the manufacturer's protocol (Life Technologies, USA). The cellular viability at day 1 was used as an indicator of the efficiency of HDF attachment. The viability between day 1 and 7 was used to evaluate the growth rate. The total number of cells was calculated using the standard curve. The growth rate was calculated using the following formula:

\section{Ln (Final cell concentration/ \\ Growth rate $\left(\mathrm{h}^{-1}\right)=\frac{\text { Initial cell concentration })}{(\text { Final culture time }- \text { Initial }}$ culture time) \\ Evaluation of HDF morphology}

Observation of HDF morphology was performed by scanning electron microscopy (SEM; FEI, USA). The OTC-I scaffolds seeded with HDF were fixed with 4\% paraformaldehyde overnight. The dehydration of fixed samples was done by immersion in a series of ethanol solutions $(30 \%, 50 \%, 70 \%$, and $100 \%$; $10 \mathrm{~min}$ each). A critical point drying machine was used to reach the critical drying point followed by sputter-coating with nanogold prior to SEM observation.

HDF were also subjected to fluorescence staining with phalloidin (Biotium, USA) to observe the actin filaments, and were also stained for alpha-smooth muscle actin ( $\alpha$-SMA) to demonstrate contractile activity. The HDF seeded on OTC-I scaffolds were fixed with $4 \%$ paraformaldehyde (Sigma) for 2 hours at room temperature. HDF were permeabilised using Triton-X100 (Sigma), and non-specific binding sites were blocked with 10\% goat serum (Sigma) for 1 hour at $37^{\circ} \mathrm{C}$. HDF were incubated with primary antibody of anti- $\alpha$-SMA (Abcam, USA) overnight at $4{ }^{\circ} \mathrm{C}$ followed by secondary antibody (Invitrogen, USA). Cells were then stained with DAPI (Invitrogen) and phalloidin for visualisation of the nucleus and filamentous actin (F-actin), respectively. Images were captured using an A1R confocal laser scanning microscope (Nikon, Japan).

\section{Statistical analysis}

All data are represented as mean \pm standard error of the mean. Statistical analysis was performed using GraphPad Prism (version 5.0). One-way analysis of variance (ANOVA) was used to compare the results of multiple groups. A $P$-value less than 0.05 was considered as significant.

\section{Results}

\section{Gross appearance of OTC-I scaffolds}

Three different scaffolds were fabricated using OTC-I. Figure 1 shows the gross appearance and ultrastructure of OTC-I film, hydrogel, and sponge, with or without crosslinking. The film was thin and translucent, while the hydrogel appeared to be semi-solid gel and translucent. In contrast, the OTC-I sponge showed a characteristic porous, opaque structure. OTC-I scaffold crosslinked with GNP exhibited a light brownish colour $\left(15^{-16)}\right.$ as compared to the non-crosslinked one (white). SEM analysis demonstrated that OTC-I film showed a contoured and rough surface, whereas collagen appeared as flakes with high porosity in an OTC-I sponge. In contrast, the OTC-I 


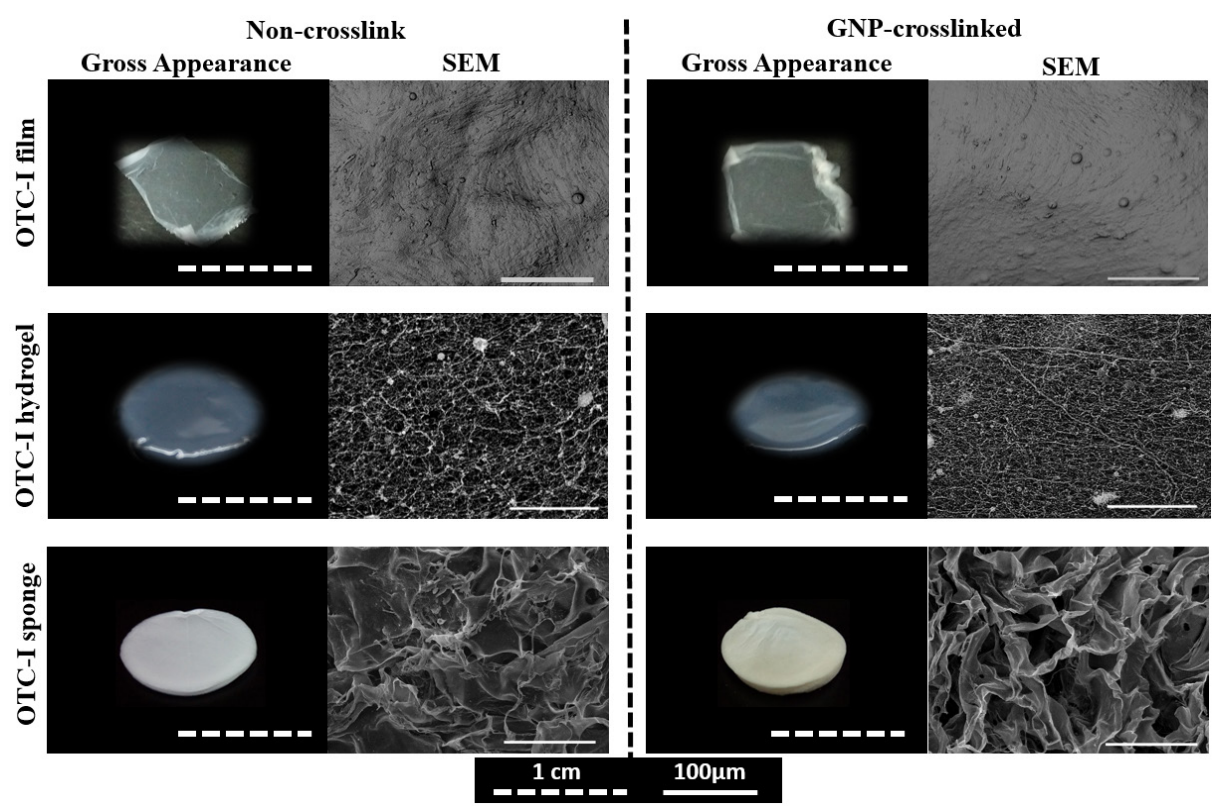

Figure 1. Gross and SEM images of OTC-I scaffolds i.e. OTC-I films, hydrogel, and sponge, with and without GNP crosslinking.

hydrogel appeared to be fibrous with medium porosity.

\section{Mechanical strength of OTC-I scaffolds}

The tensile strain and Young's modulus of OTC-I scaffolds, with or without crosslinking, are shown in Figure 2. Among the OTC-I scaffolds, sponge demonstrated a significantly higher Young's modulus and tensile strength than that of films and hydrogel. Moreover, the Young's modulus and tensile strain of OTC-I film, with or without crosslinking, was significantly higher compared to that of OTC-I hydrogel. It was also found that crosslinking of OTC-I scaffolds with GNP had no effect on the mechanical strength of the film or hydrogel. However, the mechanical strength of crosslinked OTC-I sponge was significantly higher than that of the noncrosslinked sponge.

\section{Attachment and proliferation of HDF}

HDF (biological replicates, $N=6$ and technical replicates, $n=3$ ) were seeded on a polystyrene surface (control), an OTC-I-coated surface and on top of the OTC-I scaffolds, and the efficiency of cell attachment was evaluated at 24 hours after seeding (Figure 3a). The OTC-I-coated surface and other scaffolds demonstrated significantly higher attachment of HDF compared to that on the polystyrene surface (15854 \pm 1333 cells). The crosslinked
OTC-I film exhibited the highest cell attachment (20835 \pm 686 cells) overall.

Similarly, the growth rate of HDF was significantly lower on the polystyrene surface (0.0137 $\left.\pm 0.00063 \mathrm{~h}^{-1}\right)$ compared to other conditions. Among the OTC-I scaffolds and coated surfaces, OTC-I film showed the highest growth rate $\left(0.0154 \pm 0.0011 \mathrm{~h}^{-1}\right)$ (Figure $3 \mathrm{~b}$ ).

\section{Morphological features of HDF on OTC-I-coated surface and scaffolds}

HDF seeded on the OTC-I-coated surface and scaffolds demonstrated distinct morphological features. As shown in Figure 4, HDF on the polystyrene surface showed a flat and spindle-like morphology with the presence of lamellipodia and few filopodia on day 1. However, on day 7 , it demonstrated an elongated, flat, and spindle-like morphology with long filopodia protrusions. Coating the polystyrene surface with OTC-I led to dramatic changes in HDF morphology. On day 1, HDF on the OTC-I-coated surface displayed a dendritic morphology. On day 7 , some of the HDF persisted with a similar morphology, while others were flat and polygonal with lamellipodia and numerous filopodia. In contrast, HDF on the crosslinked OTC-I-coated surface were round with prominent lamellipodia and filopodia on day 1 . However, by day $7, \mathrm{HDF}$ demonstrated morphological features similar to those on the polystyrene surface. 
Original Article | Comparative study of ovine tendon collagen scaffolds

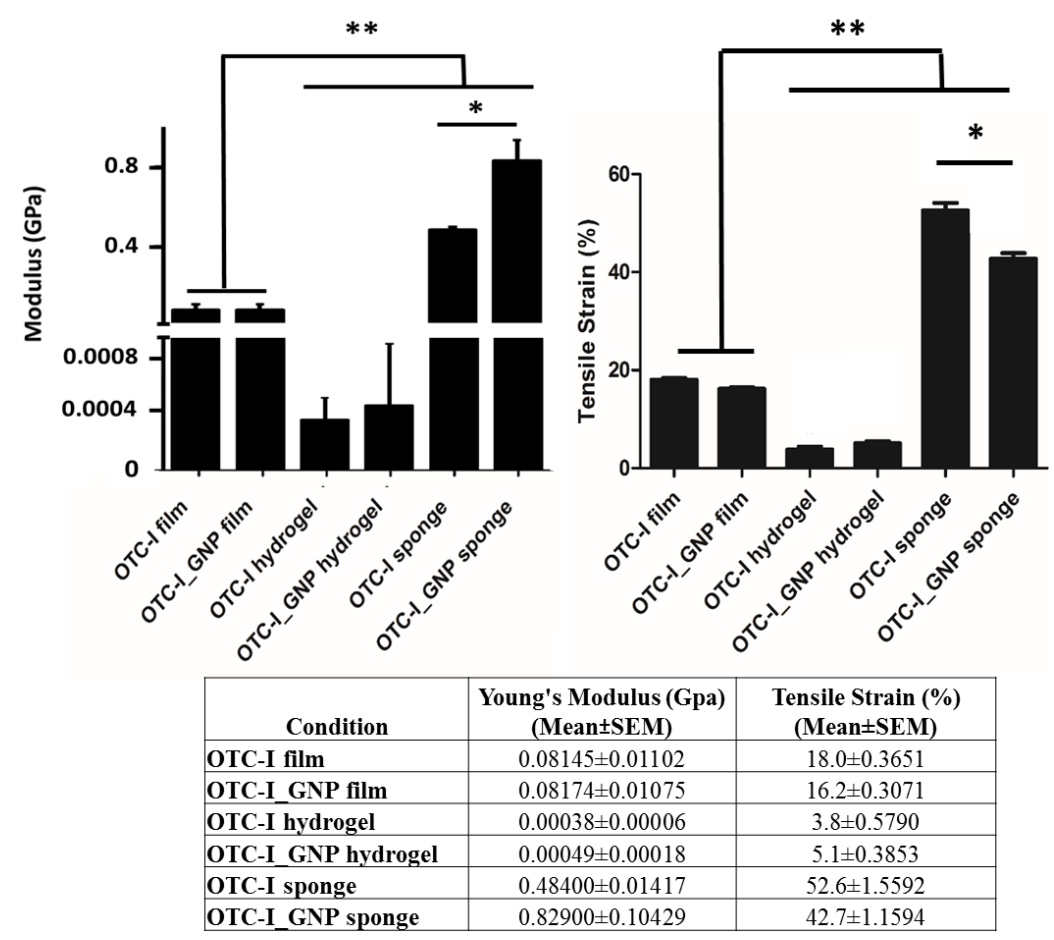

Figure 2. Mechanical strength of OTC-I scaffolds i.e. OTC-I films, hydrogel, and sponge, with and without GNP crosslinking. * Represents significant difference between crosslinked and noncrosslinked OTC-I sponge. **Represents significantly higher mechanical strength of both crosslinked and non-crosslinked OTC-I sponge compared with other scaffolds $(P<0.05$; $n=6)$.

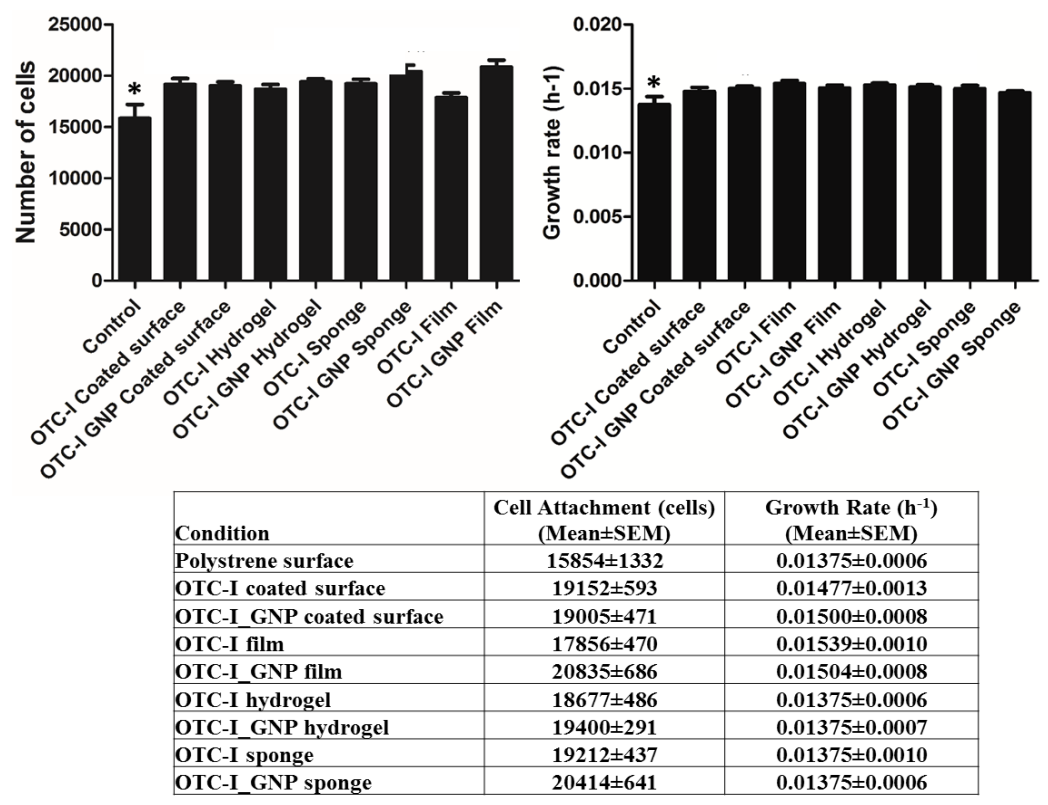

Figure 3. Human dermal fibroblast (HDF) properties under different culture conditions. a) Total number of attached HDF on coated and non-coated polystyrene surface and different OTC-I scaffolds on day 1. b) Growth rate of HDF on coated and non-coated polystyrene surfaces and different OTC-I scaffolds. *Represents significantly lower growth rate than in other conditions; OTC-I-coated surface, films, hydrogel and sponge with and without GNP crosslinking $(P<0.05 ; N=6)$. 


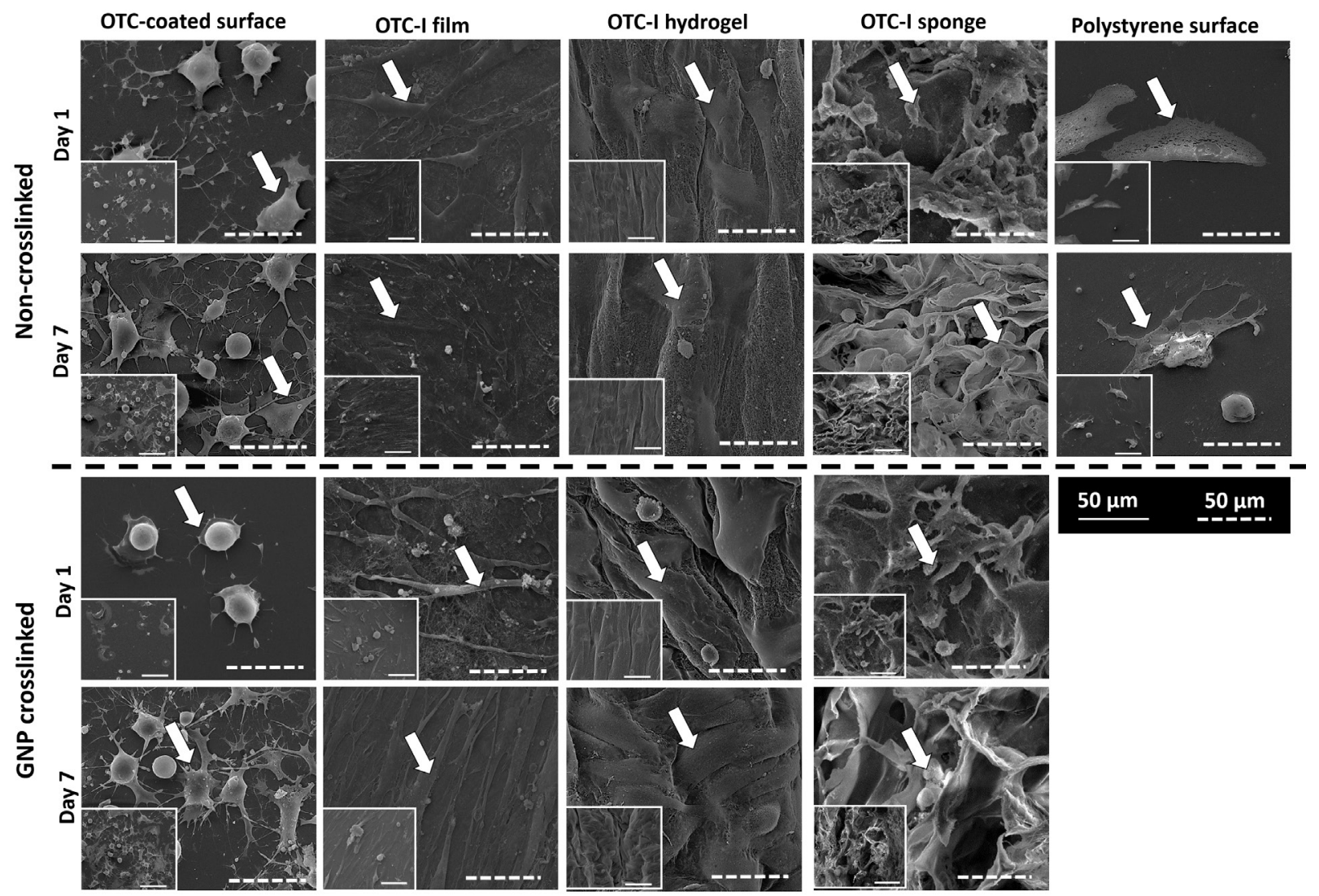

Figure 4. Representative electron micrograph image of HDF seeded on the coated and non-coated polystyrene surfaces and different OTC-I scaffolds, with or without crosslinking, on day 1 and 7.

HDF on OTC-I films showed a flat, thin, and spindle-like morphology with the presence of filopodia on day 1 . Cells became more elongated and thinner by day 7 . No changes were detected for HDF morphology on crosslinked OTC-I film. In the case of both crosslinked and noncrosslinked OTC-I hydrogels, HDF demonstrated flat, spindle-like, and spreading morphology with lamellipodia on day 1 and 7. However, protrusion of filopodia was clearly visible by day 7. In contrast, HDF on OTC-I sponges, both crosslinked and non-crosslinked, demonstrated a spindle-like morphology, although some cells were more spherical with the presence of surrounding matrices. HDF showed the presence of lamellipodia and prominent filopodia on day 1 , but were round by day 7 . However, the presence of surrounding matrices on day 7 demonstrated that HDF were viable in the OTC-I sponge.

HDF on different surfaces and scaffolds demonstrated diverse types of assembly and structure of actin cytoskeleton (Figure 5). HDF on the polystyrene surface developed actin stress fibres on day 1 , which became elongated and prominent by day 7 . In contrast, HDF on OTC-I-coated polystyrene surface developed stress fibres with shorter actin filaments than that on the polystyrene surface. As expected, no stress fibres formed in HDF when cultured on crosslinked or non-crosslinked OTC-I hydrogel. However, in a later phase of culture, HDF developed short stress fibres with filopodia on OTC-I hydrogels. In crosslinked and noncrosslinked OTC-I sponge, HDF formed short actin filaments on day 1 and 7 , which were mostly distributed along the periphery of the cells. Unlike other conditions, HDF on OTC-I film, with or without GNP crosslinking, demonstrated the formation of elongated stress fibres as early as day 1 and this was sustained until day 7 . Regarding the contractile properties of HDF (Figure 5), only HDF on OTC-I film and sponge on day 7 demonstrated the presence of a-SMA positive cells; however, the number of a-SMA positive cells was low compared to that of total cells. 
Original Article | Comparative study of ovine tendon collagen scaffolds

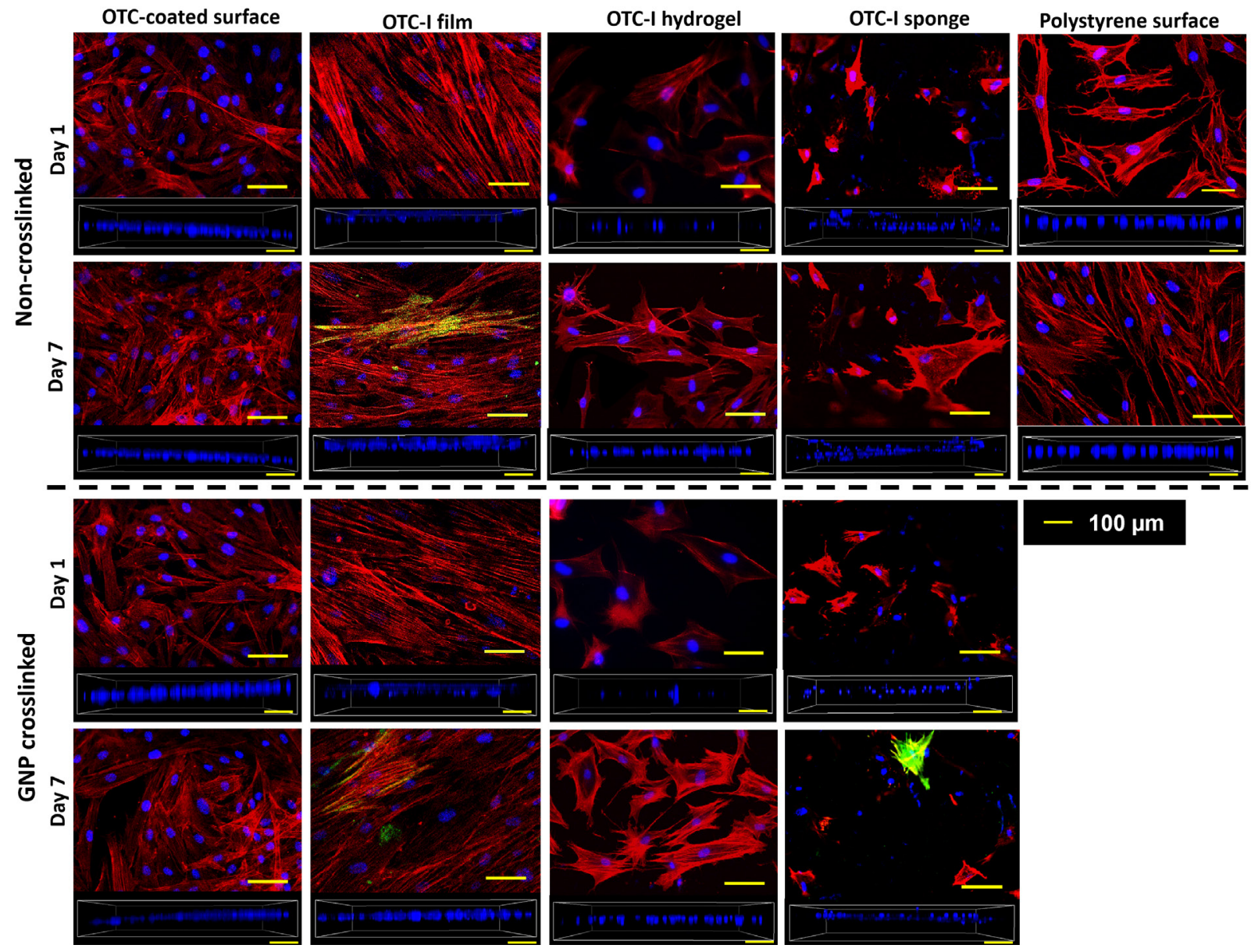

Figure 5. Fluorescence images of HDF cultured on the coated and non-coated polystyrene surfaces and different OTC-I scaffolds, with or without crosslinking, on day 1 and 7. HDF were stained for alpha smooth muscle actin (green; $\alpha$-SMA), F-actin (red; phalloidin) and nucleus (blue; DAPI). Images in the box containing the nucleis (blue; DAPI) demonstrate the distribution of the HDF in the scaffolds.

The distribution of HDF on OTC-I scaffolds was also observed by capturing three-dimensional images using confocal laser scanning microscopy. As shown in Figure 5, HDF on OTC-I sponges, both crosslinked and noncrosslinked, were distributed in different layers of the scaffolds. In contrast, HDF were located within similar layers of OTC-I film and hydrogel during the 7-day culture period.

\section{Discussion}

Collagen type I (col I) is one of the major components of extracellular matrix (ECM) in the human body and is abundant in skin, tendon, ligaments, cornea, and bone (17). Col I is regularly used as a coating material for culture surfaces to evaluate cell-ECM interactions (18).
Although it has been shown to facilitate cellular behaviours, a 2D-coated surface fails to mimic the native $3 \mathrm{D}$ physiological environment (1920). Current fabrication technology enables the moulding of col I into $3 \mathrm{D}$ scaffolds, such as film, hydrogel, and sponge, which may mimic the matrix framework of native tissues. Several comparative studies between $2 \mathrm{D}$ and $3 \mathrm{D}$ collagen matrices have demonstrated significant differences in cellular properties, such as attachment, morphology, proliferation, differentiation, and migration $(2,21-22)$. In the current study, col I isolated from ovine tendon (OTC-I) was used to fabricate $3 \mathrm{D}$ scaffolds such as film, hydrogel, and sponge. The attachment, proliferation, and morphological features of human dermal fibroblasts (HDF) on the OTC-I scaffolds were studied and compared with OTCI-coated and non-coated polystyrene surfaces. 
Col I is a commonly used natural biomaterial for tissue engineering applications because of its biocompatibility, biodegradability, and low immunogenicity $(9,11)$. Col I can be fabricated into different scaffolds including hydrogel, film, sponge and nanofibres via gelation, air drying (10), freeze-drying $(9,11)$ and electrospinning (23) techniques, respectively. These scaffolds vary in their gross appearance as well as their topographical features, porosity, and mechanical strength. These 3D scaffolds are currently being used to develop tissue substitutes for clinical applications and in vitro models as well as drug delivery vehicles.

Numerous recent studies have demonstrated that the structural features of a scaffold regulate biological properties of cells, which is crucial to form tissue substitutes $(9,11$, 24-26). HDF attachment and proliferation was significantly higher on the $3 \mathrm{D}$ OTC-I scaffolds and coated polystyrene surface compared to the non-coated polystyrene surface, but no difference was observed among the OTC-I scaffolds and coated surface. As expected, distinct morphological differences of HDF in terms of cell spreading, the formation of actin stress fibres, lamellipodia, and filopodia were observed among the OTC-I scaffolds and coated surfaces compared to the non-coated polystyrene surface, which could be due to the mechanical properties of the scaffolds and surrounding matrix environment. It has been well documented that morphological features such as cell spreading, cell area, cytoskeleton remodelling, and focal adhesion are regulated by the mechanical properties of the extracellular matrix environment (27-29). Solon et al. demonstrated that fibroblast morphology is strongly governed by the stiffness of the culture surface and surrounding matrix environments (30).

The differences in cellular morphology are mainly regulated by the assembly of actin filaments, which form protrusions (filopodia) and contractile stress fibre structures. The formation of stress fibres strongly correlates with the mechanical stress of the surrounding matrices. In general, cells demonstrate thick stress fibres when cultured on rigid substrates (such as glass or polystyrene), while these structures are thin or even absent when cultured on soft substrates $(31,32)$. The current study also demonstrated the formation of thick stress fibres on OTC-I-coated and non-coated polystyrene surfaces due to the high rigidity of the surface. Similarly, HDF on OTC-I film demonstrated thick and elongated stress fibres, which could be due to the stiffness of the film. Overlaying the film on the polystyrene surface may also contribute to this phenomenon. OTC-I sponge, which showed the highest mechanical strength among $3 \mathrm{D}$ scaffolds, displayed short but thick actin filaments, most of which were distributed along the periphery of the cells. This also explains the restricted spreading of cells in OTC-I sponge. In contrast, OTC-I hydrogel, which is soft in nature and shows very low tensile strength, demonstrated the formation of thin stress fibres. Actin cytoskeleton formation is involved in several biological processes such as attachment, growth, migration, cytokinesis, morphogenesis, etc. through signal transduction. During skin wound healing, HDF forms prominent stress fibres with contractile properties, so-called transient myofibroblasts, which speed up the process of wound closure via contraction and ECM remodelling (33). Some of the HDF on the OTC-I film and sponge expressing the a-SMA could facilitate the woundhealing process.

To improve the mechanical strength in this study, OTC-I scaffolds were crosslinked with genipin (GNP), which is a natural cross-linker. GNP has no cytotoxic effect on fibroblasts (data not shown). Crosslinking with GNP significantly increased the mechanical strength of OTC-I sponge, but had no effect on hydrogel and film scaffolds. Ultimately, no significant difference was observed between crosslinked and noncrosslinked OTC-I scaffolds and coated surface regarding morphological properties, attachment, and proliferation.

\section{Conclusion}

In conclusion, OTC-I possesses the ability to be fabricated into various $3 \mathrm{D}$ scaffolds, including film, sponge, and hydrogel. Crosslinking with GNP was shown to enhance the mechanical strength of OTC-I sponge but had no effect on film or hydrogel. Both GNP crosslinked and non-crosslinked OTC-I scaffolds were shown to be biocompatible towards HDF. This indicates that OTC-I scaffolds have potential for use in in vitro tissue models for drug discovery, and as tissue substitutes for implantation and drug delivery vehicles. Distinct differences in the morphological features of HDF were observed between the $3 \mathrm{D}$ scaffolds. This comparative study provides information on the physical and mechanical properties of OTC-I scaffolds and 
Original Article | Comparative study of ovine tendon collagen scaffolds

demonstrated the biological properties of HDF on these scaffolds. However, further studies are required to understand the association between scaffold properties and morphological differences of HDF, and their downstream effect on cellular behaviours.

\section{Acknowledgement}

The authors would like to acknowledge financial support from Ministry of Science, Technology and Innovation (MOSTI), Malaysia (Grant Code: 02-01-02-SFo964), Universiti Kebangsaan Malaysia (Arus Perdana Grant Code: AP-2013-015), and UKM Medical Centre (Fundamental Grant Code: FF-2014-370 and FF2015-087).

\section{Conflicts of Interest}

The authors declare that there is no conflict of interest.

\section{Authors' Contributions}

Conception and design: FMB, YL, RBHI, SRC

Analysis and interpretation of the data: FMB, SRC

Drafting of the article: FMB

Critical revision of the article for important intellectual content: YL, MMN, RBHI, SRC

Final approval of the article: FMB, YL, MMN, AS, RBHI, SRC

Provision of study materials or patients: MMN, AS

Statistical expertise: FMB, SRC

Obtaining of funding: RBHI, SRC

Collection and assembly of data: FMB

\section{Correspondence}

Dr Shiplu Roy Chowdhury

BSc (University of Dhaka), MSc (University of Dhaka), ME (Osaka University), PhD (Osaka University)

Tissue Engineering Centre,

Universiti Kebangsaan Malaysia Medical Centre

(UKMMC),

Jalan Yaacob Latiff, Bandar Tun Razak, 56000 Cheras, Kuala Lumpur, Malaysia

Tel: +603 91457679

Fax: +60391457678

E-mail: shiplu@ppukm.ukm.edu.my / shiplu56@ gmail.com

\section{References}

1. O'Brien FJ. Biomaterials \& scaffolds for tissue engineering. Mater. Today. 2011;14(3):88-95. https://doi.org/10.1016/S1369-7021(11)70058-X

2. Bax DV, McKenzie DR, Weiss AS, Bilek MM. The linker-free covalent attachment of collagen to plasma immersion ion implantation treated polytetrafluoroethylene and subsequent cellbinding activity. Biomaterials. 2010;31(9):25262534. https://doi.org/10.1016/j.biomaterials. 2009.12.009

3. Bin Mh Busra MF, Chowdhury SR, Bin Ismail F, Bin Saim A, Idrus RH. Tissue-engineered skin substitute enhances wound healing after radiation therapy. Adv Skin Wound Care. 2016;29(3):120-129. https://doi.org/10.1097/o1. ASW.0000480556.78111.e4

4. Zhu N, Chen X. Biofabrication of tissue scaffolds. In: Pignatello R, editor. Advances in biomaterials science and biomedical applications. InTech Crotia. 2003;315-328. https://doi. org/10.5772/54125

5. Chan BP, Leong KW. Scaffolding in tissue engineering: general approaches and tissue-specific considerations. Eur Spine $J$. 2008;17(4):467-479. https://doi.org/10.1007/ soo586-008-0745-3

6. Do AV, Khorsand B, Geary SM, Salem AK. 3D Printing of scaffolds for tissue regeneration applications. Adv Healthc Mater. 2015;4(12):1742-1762. https://doi.org/10.1002/ adhm.201500168

7. Feller L, Jadwat Y, Khammissa RA, Meyerov R, Schechter I, Lemmer J. Cellular responses evoked by different surface characteristics of intraosseous titanium implants. Biomed Res Int. 2015;2015:18. https://doi.org/10.1155/2015/171945

8. Evans NT, Irvin CW, Safranski D, Gall K. Impact of surface porosity and topography on the mechanical behavior of high strength biomedical polymers. $J$ Mech Behav Biomed Mater. 2016;59:459-473. https://doi.org/10.1016/j. jmbbm.2016.02.033 
9. Awang MA, Firdaus MA, Busra MB, Chowdhury SR, Fadilah NR, Wan Hamirul WK, et al. Cytotoxic evaluation of biomechanically improved crosslinked ovine collagen on human dermal fibroblasts. Biomed Mater Eng. 2014;24(4):1715-1724. https://doi.org/ 10.3233/BME-140983

10. Fauzi MB, Lokanathan Y, Aminuddin S, Ruszymah BHI, Chowdhury SR. Ovine tendon collagen: Extraction, characterisation and fabrication of thin films for tissue engineering applications. Mater Sci Eng C. 2016;68:163-171. https://doi.org/10.1016/j.msec.2016.05.109

11. Busra FM, Chowdhury SR, Saim AB, Idrus RB. Genotoxicity and cytotoxicity of ovine collagen on human dermal fibroblasts. Saudi Med J. 2011;32(12):1311-1312.

12. Solorio L, Zwolinski C, Lund AW, Farrell MJ, Stegemann JP. Gelatin microspheres crosslinked with genipin for local delivery of growth factors. $J$ Tissue Eng Regen Med. 2010;4(7):514-523. https://doi.org/10.1002/term.267

13. Yoo JS, Kim YJ, Kim SH, Choi SH. Study on genipin: a new alternative natural crosslinking agent for fixing heterograft tissue. Korean $J$ Thorac Cardiovasc Surg. 2011;44:197-207. https://doi.org/10.5090/kjtcs.2011.44.3.197

14. Xian LJ, Chowdhury SR, Saim AB, Idrus RBH. Concentration-dependent effect of platelet-rich plasma on keratinocyte and fibroblast wound healing. Cytotherapy 2015;17:293-300. https:// doi.org/10.1016/j.jcyt.2014.10.005

15. Bi L, Cao Z, Hu Y, Song Y, Yu L, Yang B, et al. Effects of different cross-linking conditions on the properties of genipin-cross-linked chitosan/ collagen scaffolds for cartilage tissue engineering. $J$ Mater Sci Mater Med. 2011;22(1):51-62. https://doi.org/10.1007/s10856-010-4177-3

16. $\mathrm{Mu} \mathrm{C}$, Zhang $\mathrm{K}$, Lin $\mathrm{W}$, Li D. Ring-opening polymerization of genipin and its long-range crosslinking effect on collagen hydrogel. $J$ Biomed Mater Res A. 2013;101(2):385-393. https://doi. org/10.1002/jbm.a.34338

17. Xiong X, Ghosh R, Hiller E, Drepper F, Knapp $\mathrm{B}$, Brunner $\mathrm{H}$, et al. A new procedure for rapid, high yield purification of type I collagen for tissue engineering. Process Biochem. 2009;44:1200-1212. https://doi.org/10.1016/j. procbio.2009.06.010
18. Vladkova TG. Surface engineered polymeric biomaterials with improved biocontact properties. Int J Polym Sci. 2010;2010:1-22. https://doi. org/10.1155/2010/296094

19. Geckil H, Xu F, Zhang X, Moon SJ, Demirci U. Engineering hydrogels as extracellular matrix mimics. Nanomedicine (Lond). 2010;5(3): 469484 .

20. Huh D, Hamilton GA, Ingber DE. From threedimensional cell culture to organs-on-chips. Trends Cell Biol. 2011;21(12):745-754.

21. Yamamoto A, Mishima S, Maruyama N, Sumita M. Quantitative evaluation of cell attachment to glass, polystyrene, and fibronectin- or collagen-coated polystyrene by measurement of cell adhesive shear force and cell detachment energy. $J$ Biomed Mater Res. 2000;50(2):114124. https://doi.org/10.1002/(SICI)10974636(200005)50:2<114::AID-JBM4>3.0.CO;2-6

22. Yashiki S, Umegaki R, Kino-Oka M, Taya M. Evaluation of attachment and growth of anchorage-dependent cells on culture surfaces with type I collagen coating. $J$ Biosci Bioeng. 2001;92(4):385-388. https://doi.org/10.1016/ S1389-1723(01)80244-9

23. $\mathrm{Xu} \mathrm{F}$, Sheardown $\mathrm{H}$, Hoare T. Reactive electrospinning of degradable poly(oligoethylene glycol methacrylate)-based nanofibrous hydrogel networks. Chem Commun (Camb). 2016;52(7):1451-1454. https://doi.org/10.1039/ c5cco8o53c

24. Chen G, Sato T, Ohgushi H, Ushida T, Tateishi T, Tanaka J. Culturing of skin fibroblasts in a thin PLGA-collagen hybrid mesh. Biomaterials. 2005;26(15):2559-2566. https://doi.org/ 10.1016/j.biomaterials.2004.07.034

25. Kawazoe N, Inoue C, Tateishi T, Chen G. A cell leakproof PLGA-collagen hybrid scaffold for cartilage tissue engineering. Biotechnol Prog. 2010;26(3):819-826. https://doi.org/10.1002/ btpr.375

26. Jarman-Smith ML, Bodamyali T, Stevens C, Howell JA, Horrocks M, Chaudhuri JB. Porcine collagen crosslinking, degradation and its capability for fibroblast adhesion and proliferation. $J$ Mater Sci Mater Med. 2004;15(8):925-932. https://doi.org/10.1023/ B:JMSM.0000036281.47596.cc 
Original Article | Comparative study of ovine tendon collagen scaffolds

27. Schwartz MA. Integrins and extracellular matrix in mechanotransduction. Cold Spring Harb Perspect Biol. 2010;2(12):1-13. https://doi. org/10.1101/cshperspect.aoo5066

28. Wozniak MA, Modzelewska K, Kwong L, Keely PJ. Focal adhesion regulation of cell behavior. Biochim Biophys Acta. 2004;1692(2-3):103-119. https://doi.org/10.1016/j.bbamcr.2004.04.007

29. Plant AL, Bhadriraju K, Spurlin TA, Elliott JT. Cell response to matrix mechanics: focus on collagen. Biochim Biophys Acta. 2009;1793(5):893-902. https://doi.org/ 10.1016/j.bbamcr.2008.10.012

30. Solon J, Levental I, Sengupta K, Georges PC, Janmey PA. Fibroblast adaptation and stiffness matching to soft elastic substrates. Biophys J. 2007;93(12):4453-4461. https://doi. org/10.1529/biophysj.106.101386
31. Discher DE, Janmey P, Wang YL. Tissue cells feel and respond to the stiffness of their substrate. Science 2005;310:1139-1143. https://doi. org/10.1126/science.1116995

32. Prager-Khoutorsky M, Lichtenstein A, Krishnan R, Rajendran K, Mayo A, Kam Z, et al. Fibroblast polarization is a matrix rigiditydependent process controlled by focal adhesion mechanosensing. Nat Cell Biol. 2011;13:14571465. https://doi.org/10.1038/ncb2370

33. Sandbo N, Dulin N. Actin cytoskeleton in myofibroblast differentiation: ultrastructure defining form and driving function. Transl Res. 2011;158:181-196. https://doi.org/10.1016/j. trsl.2011.05.004 